\title{
Sind die Tage des Berichtswesens
}

\section{gezählt?}

\section{Liebe Leserinnen und Leser,}

Reporting ist „das“ Spielfeld der Controller. Wie wir aus dem WHU-Controllerpanel wissen, verbringen Controller bisher mehr als einen Tag in der Woche damit. Das Spektrum reicht vom anlassgesteuerten Sonderbericht bis zum monatlichen Standardreport. Berichte sind das zentrale Mittel, um die gewünschte betriebswirtschaftliche Transparenz zu schaffen. Sie sind für einen Business Partner genauso wichtig wie für einen Controller, der sich noch sehr als Herr der Zahlen sieht. Berichte bieten immer auch die Möglichkeit zu regelmäßigem Kontakt mit dem Management, was wiederum für andere Aufgaben, z. B. Beratung des Managements, sehr hilfreich ist. Frei nach Herbert Grönemeyer: Berichte sind für Controller einfach unersetzlich.

Deshalb sollten Controller aktuell sehr hellhörig sein: Auf dem Feld des Berichtswesens zeichnen sich erhebliche Veränderungen ab, die man in der Sprache des Innovationsmanagements als „disruptiv“ bezeichnen kann. Zwei Verdrängungsbzw. Veränderungsrichtungen sind zu erkennen.

Der Begriff des „Rechnunglegens“ kommt aus einer Zeit, in der das Herstellen eines Gesamtblicks über das Geschehene sehr aufwendig war und deshalb nur selten erfolgte, im Normalfall nur zum Jahresende. Heute liegen die Daten so aufbereitet vor, dass quasi ständig ein Abschluss erstellt werden kann - selbst eine Steuerabgrenzung kann man fast tagesbezogen organisieren. Längst gibt es Monatsabschlüsse; der Weg von Abschlüssen zu tagesaktuellen Daten ist vorgezeichnet, auch wenn man sich fragen muss, ob das ein Manager wirklich braucht. Aber was ist dann noch ein Bericht?

Die andere Entwicklung betrifft den Standardcharakter von Berichten. Wenn jeder Manager die Zahlen auf seinem Dashboard selbst auswählen und gruppieren kann, wozu braucht er dann noch einen Standardbericht von seinem Controller? Dieser gibt dann im besten Fall noch die Apps vor, die auf dem Dashboard des Managers genutzt werden können. Weder macht dann noch der Begriff des Berichts Sinn, der ja suggeriert, dass dieser von einem Dritten erstellt wird (dem Manager wird berichtet), noch ist zu erwarten, dass alle Manager dieselbe Auswahl treffen. Allenfalls ist noch ein Kern denk-

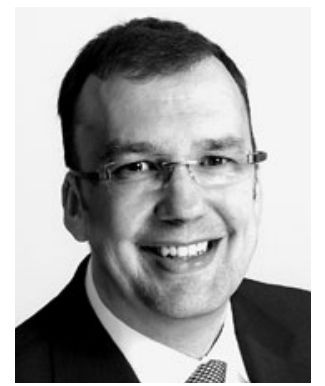

Utz Schäffer

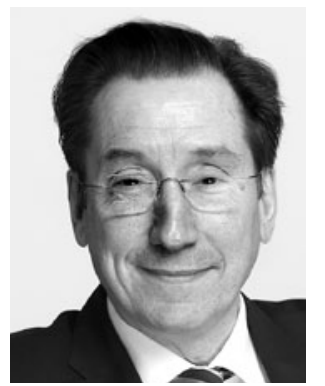

Jürgen Weber bar, den jeder Manager wählen muss, um eine babylonische Sprachverwirrung in der Kommunikation der Manager untereinander zu vermeiden.

Disruptive Veränderungen haben es an sich, dass sie bestehende Strukturen zerstören. Übertragen auf das Berichtswesen könnte dies für Controller unliebsame Folgen haben. Sie verlieren einen wesentlichen regelmäßigen Kontakt zu den Managern. Sie müssen sich darauf einstellen, dass gut informierte Manager ganz andere Informationsbedürfnisse haben als solche, die heute noch auf Berichte der Controller angewiesen sind. Sie müssen sich neue Aufgaben suchen, wenn ein Tag der Woche weitgehend frei geworden ist. Es wird spannend ...

Viel Spaß bei der Lektüre wünschen Ihnen

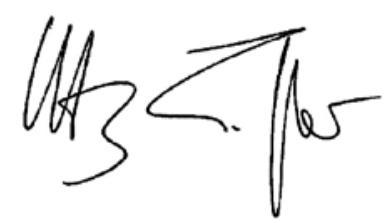

Utz Schäffer

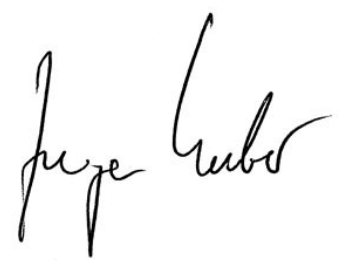

Jürgen Weber 